\title{
Sole metastatic pulmonary nodules from breast cancer simulating primary lung adenocarcinoma: Two case reports
}

\author{
KUNIHIKO MIYAZAKI $^{1}$, HIROAKI SATOH ${ }^{2}$, HIROKO WATANABE ${ }^{3}$, \\ TOSHIHIRO SHIOZAWA ${ }^{3}$, TOMOHIRO TAMURA ${ }^{2}$, MIO KAWAGUCHI $^{3}$ and NOBUYUKI HIZAWA ${ }^{3}$ \\ ${ }^{1}$ Division of Respiratory Medicine, Ryugasaki Saiseikai General Hospital, Ryugasaki, Ibaraki 301-0854; \\ ${ }^{2}$ Division of Respiratory Medicine, Mito Medical Center, Mito, Ibaraki 310-0015; ${ }^{3}$ Division of Respiratory Medicine, \\ Faculty of Medicine, University of Tsukuba, Tsukuba, Ibaraki 305-8575, Japan
}

Received June 15, 2016; Accepted August 18, 2016

DOI: $10.3892 / \mathrm{mco} .2017 .1151$

\begin{abstract}
The characteristic radiological signs of primary lung adenocarcinoma include notching, lobulation, spicular formation, pleural indentation and a bronchus leading to the nodule (bronchus sign). However, metastatic tumors rarely display such characteristics. We herein present two cases of breast cancer with sole metastatic pulmonary tumors recurring $\sim 20$ years after surgery for breast cancer. These patients exhibited radiographic signs specific to primary lung adenocarcinoma. Pulmonary metastatic nodular lesions occur through hematogenous spread; therefore, obtaining pathological specimens by transbronchial biopsy may be challenging. In our patients, however, obtaining pathological specimens by transbronchial biopsy was feasible and it ultimately confirmed the diagnosis of lung metastasis from previously treated breast cancer. To the best of our knowledge, no similar cases are reported in the English medical literature. Therefore, metastatic breast cancer may exhibit the characteristic radiological signs of pulmonary lung adenocarcinoma and, although rare, pulmonary metastasis from breast cancer should be considered even in the presence of irregularly shaped pulmonary nodule(s) following long-term disease-free survival.
\end{abstract}

\section{Introduction}

Breast cancer is one of the most common cancers in women, and the lung is one of the most prevalent sites of breast cancer metastasis $(1,2)$, which may recur after a long disease-free period following locoregional treatment $(1,2)$. We herein present the cases of 2 breast cancer patients with pulmonary metastasis that simulated primary lung adenocarcinoma.

Correspondence to: Professor Hiroaki Satoh, Division of Respiratory Medicine, Mito Medical Center, Miya-machi 2-3-7, Mito, Ibaraki 310-0015, Japan

E-mail: hirosato@md.tsukuba.ac.jp

Key words: metastatic pulmonary nodules, breast cancer, primary lung adenocarcinoma
These metastatic lesions were detected by mass screening 21 and 19 years after surgical resection of the primary breast cancers. At first, the nodules were considered as primary lung adenocarcinomas, as they shared similar radiological findings, such as irregular margins and a bronchus leading to the nodule (bronchus sign). Metastatic breast cancer may exhibit the characteristic signs of pulmonary lung adenocarcinoma. Although rare, pulmonary metastasis from breast cancer should be considered even in the presence of irregularly shaped nodule(s).

\section{Case reports}

Case 1. A 54-year-old woman was referred to our hospital in January, 2016 due to a nodule identified on a chest radiograph during a mass screening. The patient had undergone a right modified radical mastectomy for breast cancer 21 years prior. The chest CT scan revealed a nodule sized $20 \mathrm{~mm}$ with a well-defined, irregular margin in S2 of the right lung (Fig. 1) without enlargement of the mediastinal lymph nodes. The nodule exhibited notching, lobulation, spicular formation, pleural indentation, and the bronchus sign. The lesion was suspected to be either primary lung adenocarcinoma or metastatic tumor and, thus, transbronchial biopsy was performed. As the pathological results suggested that the nodule was metastatic, thoracoscopic surgery was performed. At final histology, the tumor was found to be a metastasis from breast cancer, and immunohistochemical investigation revealed that it was positive for estrogen receptor. The histopathological findings were compared with those in the surgically resected specimen 21 years prior and were found to be the same. Thereafter, the patient received systemic chemotherapy with the aromatase inhibitor letrozole, and is currently alive with stable disease.

Informed consent regarding the publication of the case details was obtained from the patient.

Case 2. A 68-year-old woman was referred to our hospital in November, 2015 due to three pulmonary nodules identified on a chest radiograph taken during a mass screening. The patient had undergone a left radical mastectomy for breast cancer 19 years prior. The chest CT scan revealed a 28 -mm nodule in 


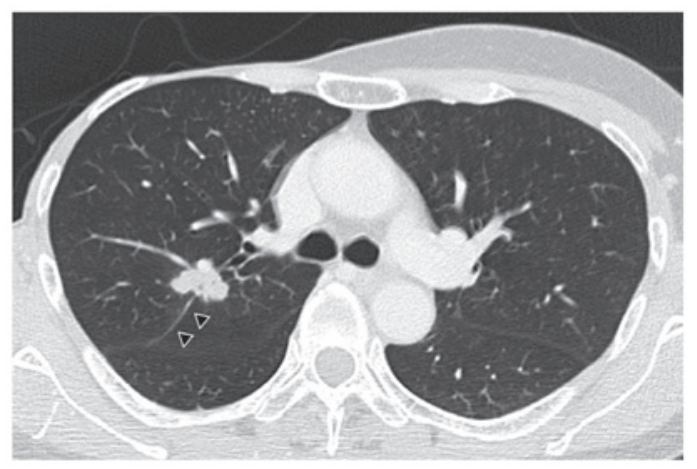

Figure 1. The chest computed tomography scan revealed a 20 -mm nodule with a well-defined, irregular margin in S2 of the right lung. The nodule exhibited notching, lobulation, (arrowheads), specular formation, pleural indentation (arrows), and a bronchus leading to the nodule (bronchus sign).
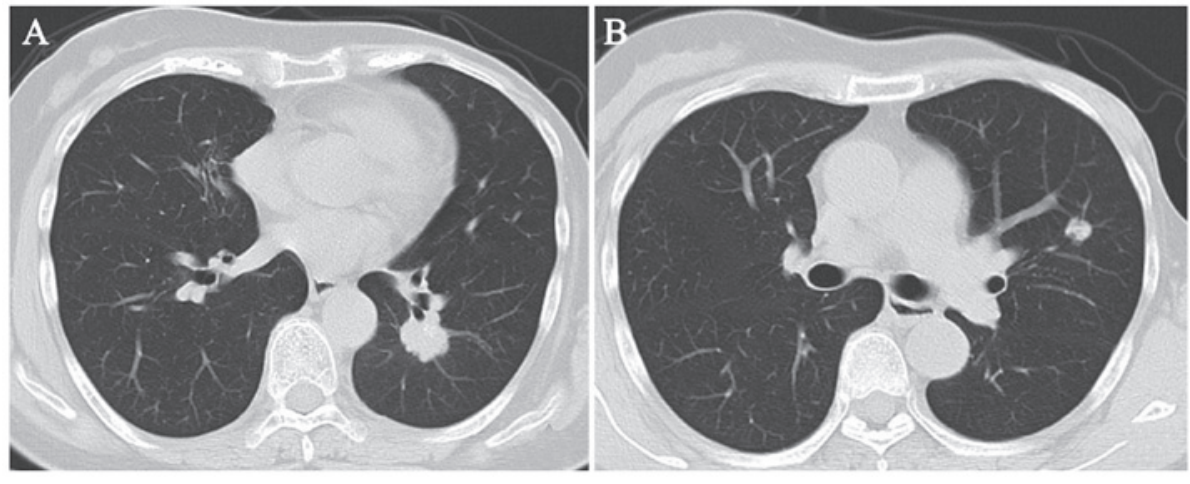

Figure 2. The chest computed tomography scan revealed (A) a 28-mm nodule in left lower lobe (arrow), a 21-mm right lingular nodule (not shown) and (B) a 21-mm nodule in the left upper lobe (arrow).

the left lower lobe, a 21-mm nodule in the left upper lobe, and a 21-mm right lingular nodule (Fig. 2), without enlargement of the mediastinal lymph nodes. The nodules exhibited notching and the bronchus sign. Primary lung adenocarcinoma or metastatic tumor was suspected and, thus, transbronchial biopsy of the lingular nodule was performed. At final histology, the lesion was found to be a metastasis from breast cancer, and immunohistochemical investigation revealed that the tumor was positive for estrogen and progesterone receptors. Thereafter, the patient received systemic chemotherapy with the aromatase inhibitor anastrozole, and is currently alive with stable disease.

Informed consent regarding the publication of the case details was obtained from the patient.

\section{Discussion}

Breast cancer is one of the most common cancers in women and may be associated with various thoracic manifestations, either from treatment, its complications, or tumor recurrence and metastasis (3). Lymphangitic metastasis, multiple nodules and a solitary nodule are the most frequently observed patterns of pulmonary metastasis from breast cancer (1-4). Lymphangitic lung metastasis of breast cancer usually appears as reticular or reticulonodular interstitial markings, or interlobar septal thickening (Kerley B lines) on imaging (3). Other patterns of pulmonary metastasis, such as a solitary or multiple pulmonary nodules, occur by means of hematogenous spread. The presence of lung metastasis is a major factor when considering treatment options for breast cancer. Thus, the accurate diagnosis of thoracic manifestations in patients with breast cancer is crucial.

Solitary or multiple pulmonary nodules are generally spherical or ovoid, vary in size, are sharply demarcated, and are located mostly in the periphery of the lung (5-8). In primary lung adenocarcinoma, notching and irregularity of the nodule margins is commonly observed, whereas a smooth margin is a sign of metastatic tumor, which results from hematogenous tumor spread. Pleural indentation and the bronchus sign are also known to be characteristic signs of primary lung adenocarcinoma on CT scan.

Development of a solitary pulmonary nodule in patients previously treated for breast cancer may represent something other than recurrent disease. Casey et al (9) found that 52\% of breast cancer patients presenting with a solitary pulmonary nodule had primary lung cancer, $43 \%$ had metastatic breast cancer and 5\% had benign lesions. Histological confirmation is required for correct diagnosis and treatment and lung biopsy is considered when there are unexplained signs and symptoms with atypical radiographic characteristics that are usually progressive or rapidly deteriorating. According to Fujii et al, there are few reported cases of cancer recurrence after $>15$ years of disease-free survival following locoregional treatment of breast cancer (10). That study reported a case of lung metastasis from breast cancer after an 18-year disease-free interval (10). Tomita et al reported a case of pulmonary metastasis from breast cancer following an 18-year disease-free 
interval that responded to tamoxifen treatment (11). In our cases, both patients presented with cancer recurrence after $>15$ years of disease-free survival following locoregional treatment of breast cancer. Although very rare, development of a sole pulmonary metastasis after a long-term interval from the diagnosis of breast cancer is possible. The precise mechanism underlying cancer recurrence after long-term disease-free survival has not been fully elucidated.

Of note, in our patients, the metastatic lesions were irregularly shaped nodules. These radiographic findings were clearly different from those of pulmonary metastasis from breast cancer, and similar to those of primary lung adenocarcinoma. To the best of our knowledge, a similar case has not been reported in the English medical literature to date. Based on the present study, we recommend that metastatic lesion from breast cancer be considered in the differential diagnosis of a solitary or multiple pulmonary nodules with irregularly shaped margins and vascular convergence, particularly in patients with breast cancer and those with a history of breast cancer.

\section{References}

1. Kreisman H, Wolkove N, Finkelstein HS, Cohen C, Margolese R and Frank H: Breast cancer and thoracic metastases: Review of 119 patients. Thorax 38: 175-179, 1983.
2. Kamby C, Vejborg I, Kristensen B, Olsen LO and Mouridsen HT: Metastatic pattern in recurrent breast cancer. Special reference to intrathoracic recurrences. Cancer 62: 2226-2233, 1988.

3. Jung JI, Kim HH, Park SH, Song SW, Chung MH, Kim HS, Kim KJ, Ahn MI, Seo SB and Hahn ST: Thoracic manifestations of breast cancer and its therapy. Radiographics 24: 1269-1285, 2004.

4. Ohnishi H, Haruta Y, Yokoyama A, Nakashima T, Hattori N and Kohno N: Metastatic breast cancer presenting as air-space consolidation on chest computed tomography. Intern Med 48: 727-731, 2009

5. Herold CJ, Banier AA and Fleischmann D: Lung metastasis. Eur Radiol 6: 596-606, 1996.

6. Davis SD: CT evaluation for pulmonary metastases in patients with extrathoracic malignancy. Radiology 180: 1-12, 1991.

7. Connolly JE Jr, Erasmus JJ and Patz EF Jr: Thoracic manifestations of breast carcinoma: Metastatic disease and complications of treatment. Clin Radiol 54: 487-494, 1999.

8. Seo JB, Im J, Goo JM, Chung MJ and Kim MY: Atypical pulmonary metastases: Spectrum of radiologic findings. Radiographics 21: 403-417, 2001.

9. Casey JJ, Stempel BG, Scanlon EF and Fry WA: The solitary pulmonary nodule in the patient with breast cancer. Surgery 96: 801-805, 1984

10. Fujii T, Yajima R, Yamaki E, Kohsaka T, Yamaguchi S, Tsutsumi S, Mogi A, Asao T and Kuwano H: Pulmonary metastasis from breast cancer with an 18-year disease-free interval: Implication of the role of surgery. Int Surg 97: 281-284, 2012.

11. Tomita M, Matsuzaki Y, Edagawa M, Maeda M, Shimizu T, Hara M, Yamamoto A and Onitsuka T: A case of pulmonary metastasis from breast cancer following an 18-year disease-free interval that responded to tamoxifen treatment. Breast Cancer 9: $82-85,2002$. 